\title{
Combined backscatter and transmission method for nuclear density gauge
}

\author{
Seyed Mohammad Golgoun ${ }^{1, a}$, Dariush Sardari ${ }^{1}$, Mahdi Sadeghi ${ }^{2}$, Mohammad Ebrahimi ${ }^{3}$, Mojtaba Aminipour ${ }^{4}$ and \\ Mohammad Reza Davarpanah ${ }^{5}$ \\ ${ }^{1}$ Islamic Azad University, Science and Research Branch, Department of Medical Radiation, P.O. Box 14515-775, Tehran, Iran \\ ${ }^{2}$ Nuclear Science and Technology Research Institute, Radiation Application Research School, Tehran, Iran \\ ${ }^{3}$ Sharif University of Technology, Department of Energy Engineering, P.O. Box 11365-8639, Tehran, Iran \\ ${ }^{4}$ Amirkabir University of Technology, Department of Energy Engineering and Physics, P.O. Box 15875-4413, Tehran, Iran \\ ${ }^{5}$ Pars Isotope Co., P.O. Box 14376-63181, Tehran, Iran
}

\begin{abstract}
Nowadays, the use of nuclear density gauges, due to the ability to work in harsh industrial environments, is very common. In this study, to reduce error related to the $\rho$ of continuous measuring density, the combination of backscatter and transmission are used simultaneously. For this reason, a ${ }^{137} \mathrm{Cs}$ source for Compton scattering dominance and two detectors are simulated by MCNP4C code for measuring the density of 3 materials. Important advantages of this combined radiometric gauge are diminished influence of $\mu$ and therefore improving linear regression.
\end{abstract}

\section{Introduction}

These days, practically every industry uses radiation in some way. Science and industry use radioisotopes in a variety of ways to improve productivity and, in some cases, to gain information that cannot be obtained in any other way. Nuclear techniques are increasingly used in science, industry and environmental management. The continuous analysis and rapid response of nuclear techniques, many involving radioisotopes, mean that reliable flow and analytic data can be constantly available. This results in reduced costs with increased product quality.

Although scientists have only known about radiation since the 1890s, they have developed a wide variety of uses for this natural phenomenon. Today, to benefit humankind, radiation is used in veterinary, medicine, academics, and industry, as well as for generating electricity. In addition, radiation has useful applications in such areas as agriculture, archaeology (carbon dating), geology (mining and aggregates) and many others.

\section{Theoretical Principles}

The radiometric density gauge is designed for continuous measurement of the density of liquids, suspensions, slurries of materials. Measurement is made without physical contact and is unaffected by changes of pressure, flow rate and viscosity. A nuclear gauge is a tool that consists of a radioactive source and a detector. The source emits a directed beam of particles and a detector would receive this beam. The radiation that comes from a radioisotope has its intensity reduced by matter between the radioactive source and a detector which is used to measure this reduction. This principle can be used to gauge the presence or the absence, or even to measure the quantity, density, thickness and moisture of material. The beam of Gamma rays emitted by a radioactive source, generally ${ }^{137} \mathrm{Cs}$ or ${ }^{60} \mathrm{Co}$ (depending on application), passes through the testing material to $\mathrm{NaI}$ detector, which converts it into output in the form of pulse rate. The strength of the pulse rate, in counts per minute $(\mathrm{cpm})$ depends upon the activity of the source, on a geometric layout and on the quantity of material through which the rays have passed. It will be a function of the density of the processing material so long as the volume and geometric disposition of the material remains constant

In this study we used collimated point ${ }^{137} \mathrm{Cs}$ because it emits gamma photons of initial energy of $662 \mathrm{keV}$. In simulation we considered isotropic radiations and for the ${ }^{137} \mathrm{Cs}$ source energy, Compton scattering is the dominant interaction [1,8]. Both photoelectric effect and pair production have mass attenuation coefficients that are heavily dependent on elemental composition that is why only those source energies within the "Compton window" are useful for densitometry. In the MCNP4C simulation that will be discussed later, we selected transmission configuration and simulated pipe with three different materials individually [2].

a e-mail: Saygoldens@gmail.com 


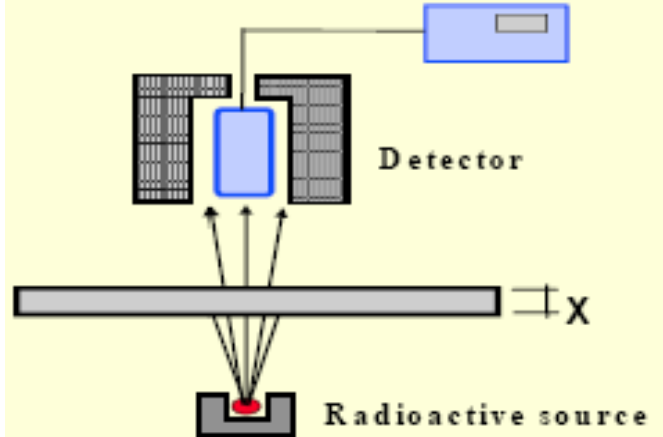

Figure 1. Principle of transmission method [7].

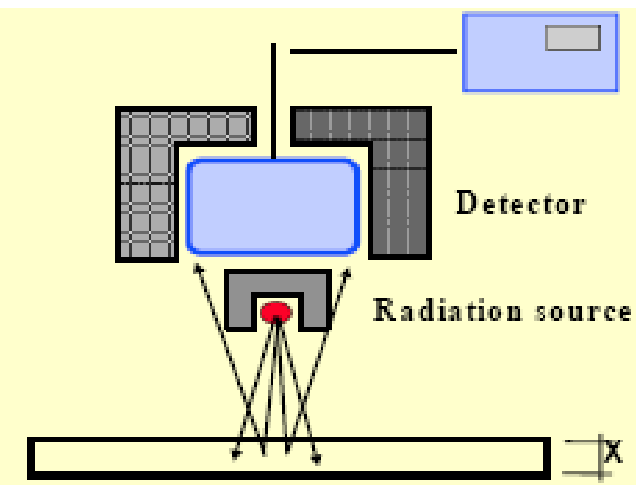

Figure 2. Backscatter method [7].

The nuclear density gauge operates on the principle that gamma ray is absorbed as a function of density expressed mathematically (without buildup factor consideration) as $[1,4,8]$ :

$$
I_{t}=I_{0} e^{-\mu \rho x}
$$

Where $I_{t}$ transmitted radiation intensity, $I_{o}$ intensity measured when no material is present, $\mu$ total mass absorption coefficient of material, $\rho$ density of material, $x$ thickness (distance between the source and detector).

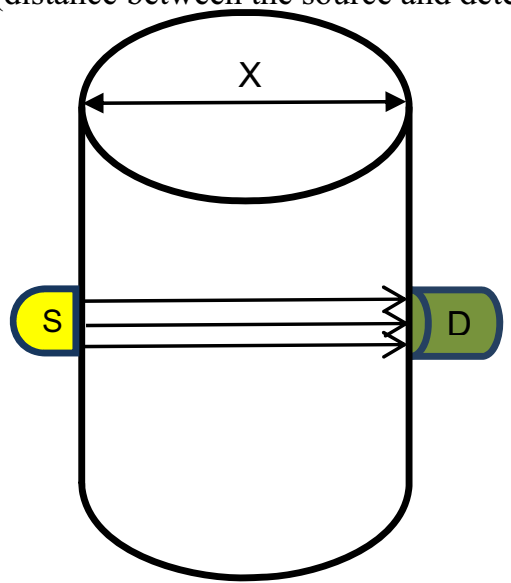

Figure 3. Transmission density gauge.
Table 1. Material characteristics.

\begin{tabular}{|c|c|c|c|c|}
\hline $\begin{array}{c}\text { Atomic } \\
\text { element }\end{array}$ & $\begin{array}{c}\text { Atomic } \\
\text { symbol }\end{array}$ & $\begin{array}{c}\text { Atomic } \\
\text { No }(\mathrm{Z})\end{array}$ & $\begin{array}{c}\text { Mass } \\
\text { No }(\mathrm{A})\end{array}$ & $\mathrm{Z} / \mathrm{A}$ \\
\hline Oxygen & $\mathrm{O}$ & 8 & 16.00 & 0.500 \\
\hline Silicon & $\mathrm{Si}$ & 14 & 28.09 & 0.4984 \\
\hline Aluminum & $\mathrm{Al}$ & 13 & 26.98 & 0.4818 \\
\hline Iron & $\mathrm{Fe}$ & 26 & 55.85 & 0.4655 \\
\hline Calcium & $\mathrm{Ca}$ & 20 & 40.08 & 0.4990 \\
\hline Sodium & $\mathrm{Na}$ & 11 & 22.99 & 0.4785 \\
\hline Potassium & $\mathrm{K}$ & 19 & 39.10 & 0.4895 \\
\hline Magnesium & $\mathrm{Mg}$ & 12 & 24.31 & 0.4936 \\
\hline Hydrogen & $\mathrm{H}$ & 1 & 1.008 & 0.9921 \\
\hline Water & $\mathrm{H}_{2} \mathrm{O}$ & 10 & 18.016 & 0.5551 \\
\hline
\end{tabular}

The density of a material is dependent upon its atomic mass (A), but the count rate in the nuclear density gauge is dependent on the number of electrons (atomic number $(Z)$ ). As above table, for the most materials $A=2 Z$ [1].

Because the source to detector distance is fixed, ' $\mathrm{X}$ ' in eq. 1 is constant. If the experiment source is ${ }^{137} \mathrm{Cs}$ with energy of $662 \mathrm{keV}$ then for variant materials the mass absorption coefficient relationship is:

$$
\mu_{2}=\mu_{1}\left(\frac{A_{1}}{A_{2}}\right)\left(\frac{Z_{2}}{Z_{1}}\right)
$$

by considering $\mathrm{A}=2 \mathrm{Z}$ then

$$
\frac{\mu_{2}}{\mu_{1}}=1
$$

It means that mass absorption coefficient ' $\mu$ ' is constant for a given process material. Therefore, the resultant radiation, ' $\mathrm{I}_{\mathrm{t}}$ ' is only a function of process density ' $\rho$ '.

By transforming equation (1) to the logarithmic form, we can write the equation (1) in terms of $\rho$ :

$$
\begin{aligned}
& \operatorname{Ln}\left(I_{t}\right)=\operatorname{Ln}\left(I_{0}\right)-\mu \rho x \\
& \rho=\frac{\operatorname{Ln}\left(I_{t}\right)-\operatorname{Ln}\left(I_{0}\right)}{\mu x} \\
& \rho=\frac{1}{\mu x} \operatorname{Ln}\left(I_{t}\right)-\frac{\operatorname{Ln}\left(I_{0}\right)}{\mu x}
\end{aligned}
$$

Due to constant consideration of $\mu$, equation (6) means that there is a linear relationship between $\rho$ and $\operatorname{Ln}\left(I_{t}\right)$ and therefore the equation (7) will be written:

$$
\rho=C_{1} \operatorname{Ln}\left(I_{t}\right)+C_{2}
$$

that $\mathrm{C}_{1}$ and $\mathrm{C}_{2}$ are constants.

\section{The Method}

All relations above are for density gauge with both source and detector collimated. In this configuration the buildup will be reduced by both source and detector shielding that we consider this as external buildup reduction. 


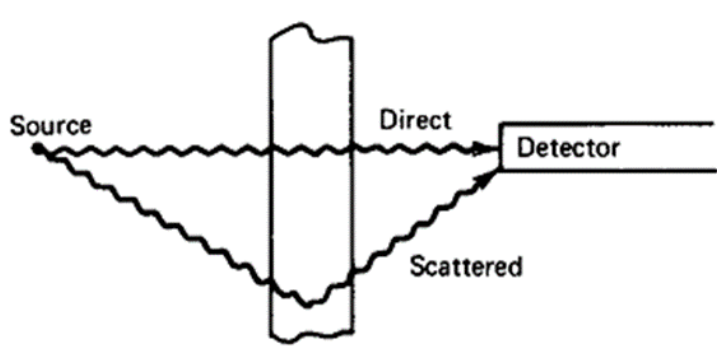

Figure 4. Principle of buildup effect [8].

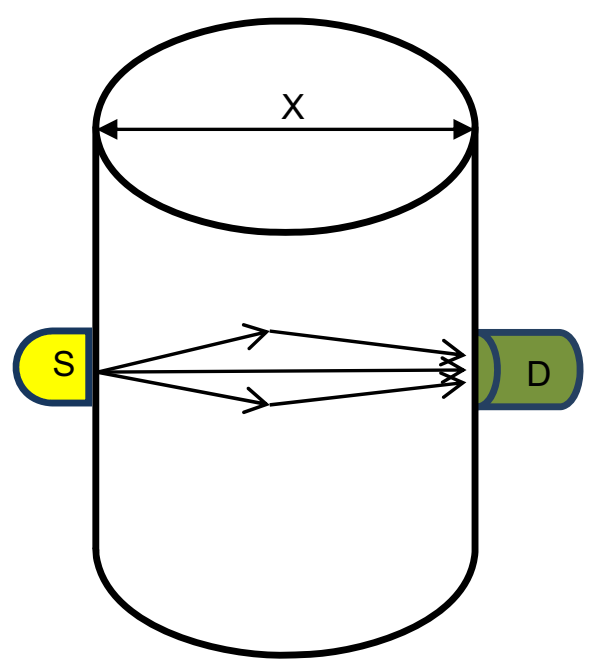

Figure 5. Simulated density gauge without internal buildup reduction.

But in our research we used new configuration for buildup reduction that is coincidence method buildup reduction. We mentioned this method as an online internal buildup reduction. As shown in Fig. 6 we used 2 detectors that could work in coincidence mode for online simultaneous buildup reduction.

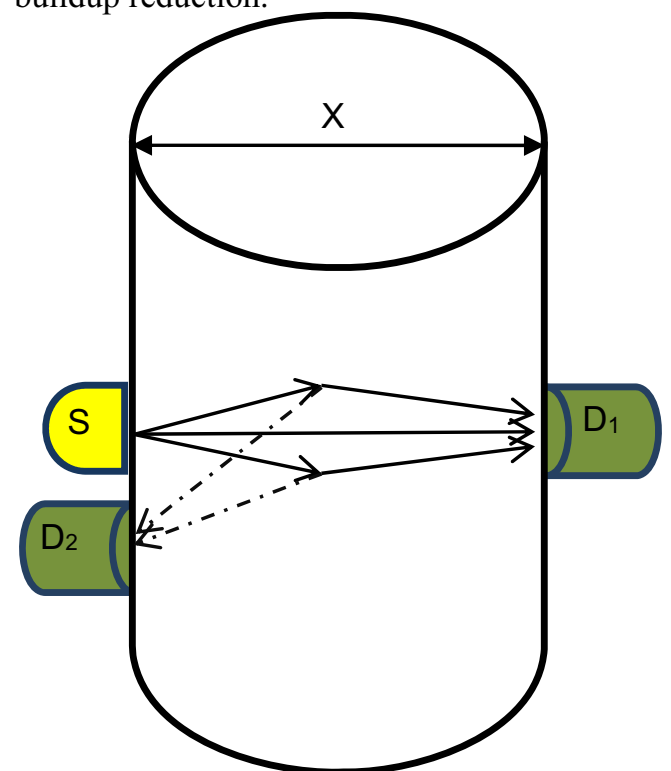

Figure 6. Simulated density gauge with internal buildup reduction.
Detector number1 would receive transmission intensity plus buildup and the detector number 2 could receive just buildup resulting from gamma interactions with matter. Buildup factor is described as $[3,8]$ :

$$
B=\frac{I_{\text {total }}}{I_{\text {unscattered }}}
$$

So the transmission equation considering buildup would be:

$$
I_{t}=B I_{0} e^{-\mu \rho x}
$$

similar to equation (1) we transformed equation (9) to logarithmic form and then we get:

$$
\operatorname{Ln}\left(I_{t}\right)-\operatorname{Ln}(B)=\operatorname{Ln}\left(I_{0}\right)-\mu \rho x
$$

$B$ in above equation is a function of $\rho$, so there is no linear relationship between $\operatorname{Ln}\left(I_{t}\right)$ and $\rho$. Therefore we estimate $\mathrm{B}$ from backscattered radiation by adding another detector to the source side (detector No. 2). If we consider to Fig. 6 again, it is clear that, this configuration is combination of backscatter and transmission methods for measuring the density. Thereupon, the buildup factor formula would change to:

$$
B=\frac{I_{D 1}}{I_{D 1}-I_{D 2}}
$$

where $I_{D 1}$ transmitted radiation intensity to the detector number 1 and $I_{D 2}$ backscattered radiation to the detector number 2 . In the equation (10) B was one of variables. So we can put equation (11) instead of B in equation (10):

$$
\operatorname{Ln}\left(I_{D 1}-I_{D 2}\right)=\operatorname{Ln}\left(I_{0}\right)-\mu \rho x
$$

above equation can be arranged on the basis of $\rho$ :

$$
\rho=\frac{1}{\mu x} \operatorname{Ln}\left(I_{D 1}-I_{D 2}\right)-\frac{\operatorname{Ln}\left(I_{0}\right)}{\mu L}
$$

Now equation (13) is a linear relationship between $\rho$ and $\operatorname{Ln}\left(I_{D 1}-I_{D 2}\right)$.

\section{MCNP4C simulation}

There are two main procedures of calculating density of the matter with known density materials. First method is point calibration that uses one, two or more calibration points. Second method is curve fit that calculates calibration equation for calibration points. In this study we used point calibration and MCNP4C code for simulation of counting system for three arbitrary testing materials. The materials are gasoline, gas oil and pure water with the known density of $0.71 \mathrm{gr} / \mathrm{cm}^{3}, 0.83 \mathrm{gr} / \mathrm{cm}^{3}$ and $1 \mathrm{gr} / \mathrm{cm}^{3}$, respectively. The radioactive point source is ${ }^{137} \mathrm{Cs}$ and the emitted beam to the detector is narrow $[5,6]$. The detectors are 2 inch $\mathrm{NaI}$ detector that could receive both scattered and transmitted radiations. The source to detector number 2 distance is $10 \mathrm{~cm}$ and the simulated iron pipe has $14 \mathrm{~cm}$ inner diameter and $14.5 \mathrm{~cm}$ outer diameter. We calculated linear regression for both combined method and transmission method, and then made a comparison at these 2 methods. 


\section{Conclusions}

In some special industries those their productions have close density ranges, it is very important to calculate density in reliable manner. For example in oil industry the density of gasoline ranges from $0.71-0.77 \mathrm{~g} / \mathrm{cm} 3$. It is important to note that in transmission method without buildup reduction that simulated by MCNP4C tool, we supposed that the radiation beam is in optimistic condition (directed beam), so in fact, the regression in Fig. 5 is not reachable and the regression without buildup correction would be worse than 0.9736 in real configuration. With the simulation of combined backscatter and transmission density meter, linear regression of the density of three testing materials has improved by $2.4 \%$.

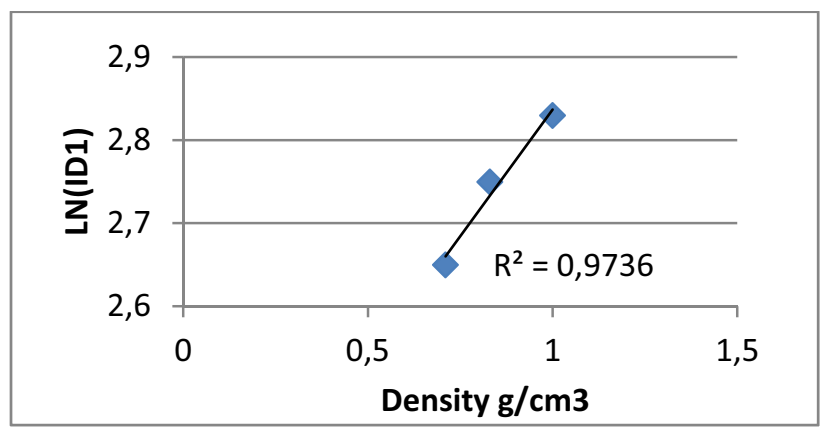

Figure 7. Diagram between density and logarithmic intensity (without internal reduction).

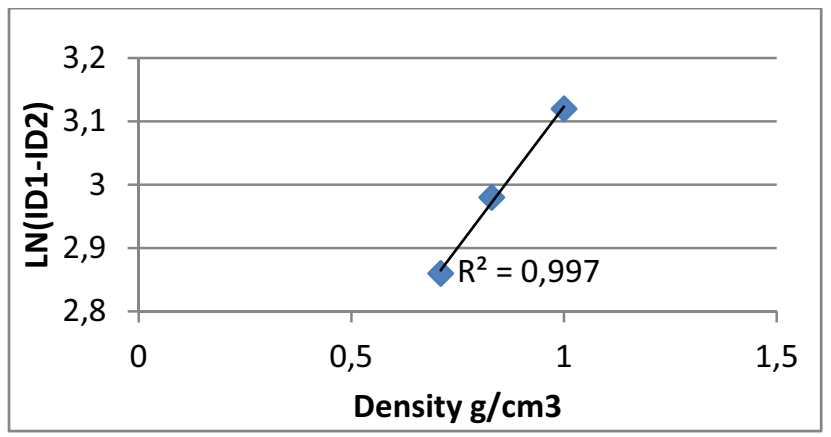

Figure 8. Influence of internal buildup correction.

\section{Acknowledgment}

We appreciate and acknowledge support for work on this article by Pars Isotope Co.

\section{References}

1. S.A. Tan and T.F. Fwa. Ndt\&E Int. 24 (1991)

2. A. Vidal, G. Viesti, F. Pino, H. Barros, L. Sajo-

Bohus. EPJ Web of Conferences. 66 (2014)

3. Y. Haiuma. Radiat. Phys. Chem. 41 (1993) 631

4. B.D. Sowerby, C.A. Rogers. Appl. Radiat. Isotopes 63 (2005) 789

5. E.R Christensen. Nucl. Eng. Des. 24 (1973) 431

6. D. Sardari, S. Saudi, M. Tajik. Ann. Nucl. Energy 38 (2011) 628

7. IAEA-TECDOC-1459. (2005) ISBN 92-0-107805-6

8. G.F. Knoll. Radiation Detection and Measurement $3^{\text {rd }}$ ed. (1989) ISBN 0-471-07338-5. Wiley, New York 